\title{
An environmentally friendly carbon material and its composite with MnO2 as an electrode material for a supercapacitor
}

\author{
Tianliang Li, Yanwei Chen, Yilin Liu \& Sheng Han* \\ Shanghai institute of technology, Fengxian, Shanghai, China
}

\begin{abstract}
An environment-friendly carbon material with an atomic layer of graphene oxide (GO)-graphene and an atomic layer of GO, hereafter denoted as GO-G-GO, was fabricated by an easy one-step hydrothermal method. GO-G-GO was obtained by centrifugation to separate GO and graphite from the remainder of products of a modified Hummers method by which GO was produced. A composite of our new material with $\mathrm{MnO} 2$ was also prepared. The structure, composition, and electrochemical properties of MnO2/reduced GO-G-GO composites and GO-G-GO were investigated by XRD, CV, SEM, and galvanostatic charge-discharge curves. MnO2/RFLG composites (62.4wt\% MnO2) exhibited a high specific capacitance of $140 \mathrm{~F} / \mathrm{g}$ in $1 \mathrm{M}$ $\mathrm{Na} 2 \mathrm{SO} 4$ at a current density of $1 \mathrm{~A} / \mathrm{g}$ after 1000 cycles.
\end{abstract}

\section{INTRODUCTION}

Graphene with a single-layer structure, high strength, and high electrical conductivity is an excellent material for energy storage and conversion. ${ }^{[1-3]}$ Many studies have been conducted on graphene or graphene oxide (GO)-based composites with metal and metal oxides. Remarkable success has been achieved, and these materials may be industrially produced in the near future. However, the high costs of producing graphene and GO hinder their commercial-scale manufacture and applications.

GO used in experiments is mostly produced by a modified Hummers method, CVD, and mechanical separation from natural graphite ${ }^{[4]}$. GO produced by a modified Hummers method is widely used for its single-layer structure and easy dispersion in water or other organic solutions. A modified Hummers method has potential future for commercial-scale manufacture because it is easy to execute and low cost. However, the efficiency is low. Improving its efficiency is difficult, but few researchers have focused on using the remainder of products of a modified Hummers method.

This work aimed to study the remainder of products of a modified Hummers method and found that these products comprised GO, graphite, and materials mostly consisting of a few layers of graphene with an atomic layer of GO-graphene and an atomic layer of GO. This material is hereafter denoted as GO-G-GO and is shown in Fig. 1(a). GO-G-GO had higher conductivity than $\mathrm{GO}$ because of the existence of a middle layer of graphene.

$\mathrm{MnO}_{2}$ is a potential material for energy storage and conversion because of its high theoretical density, low cost, environment friendliness, and natural abundance ${ }^{[5,6]}$. However, the poor conductivity of $\mathrm{MnO}_{2}\left(10^{-5} \sim 10^{-6} \mathrm{~S} / \mathrm{m}\right)$ hinders its potential as an excellent material for supercapacitors. ${ }^{[7-9]}$

To improve the electrical conductivity and broaden the application of $\mathrm{MnO}_{2}$, it is widely studied for use in $\mathrm{MnO}_{2}$-based electrical supercapacitors (ECs). Recently, thin $\mathrm{MnO}_{2}$ layers have been loaded onto high-conductivity materials such as metal, ${ }^{[10]}$ conducting polymer, ${ }^{[11,12]}$ carbon nanotube, ${ }^{[13,14]}$ or graphene ${ }^{[15]}$ for high performance. In all abovementioned cases, the materials used are high cost or the method is complex.

To realize the commercial application of $\mathrm{MnO}_{2}$-based ECs, novel $\mathrm{MnO}_{2} /$ reduced GO-G-GO $\left(\mathrm{MnO}_{2} / \mathrm{R}-\mathrm{GO}-\mathrm{G}-\mathrm{GO}\right)$ sandwich structured materials were designed and synthesized in this work. The middle layer of RFLG was made of GO-G-GO reduced by a hydrothermal method. In such a design, the middle multi-layer graphene provides an electron "superhighway" for charge storage and delivery because of its high conductivity. Thus, this material had higher conductivity than GO.

A one-step hydrothermal method was then used to prepare $\mathrm{MnO}_{2} / \mathrm{R}-\mathrm{GO}-\mathrm{G}-\mathrm{GO}$ composites as novel electrode materials. GO-G-GO contained as many $\mathrm{C}=\mathrm{O},-\mathrm{COOH}$, and $\mathrm{C}-\mathrm{OH}$ groups as $\mathrm{GO}$. 
Thus, our material had a large surface for the deposition of $\mathrm{MnO}_{2}$ and provided perfect conductivity of the electrode because of the middle layer of graphene. Our experimental results showed a specific capacitance of $140 \mathrm{~F} / \mathrm{g}$ at a current density of $1 \mathrm{~A} / \mathrm{g}$ in $1 \mathrm{M} \mathrm{Na}_{2} \mathrm{SO}_{4}$ after 1000 cycles.

\section{EXPERIMENTAL SECTION}

\subsection{Synthesis of $G O-G-G O$}

GO-G-GO was collected from the remainder of products of a modified Hummers method. ${ }^{\text {[16] }}$ About $30 \mathrm{~g}$ of the remainder (10\% dried materials) was added to $1000 \mathrm{ml}$ of deionized water and supersonicated for $3 \mathrm{~h}$. Then, the suspension was concentrated at $500 \mathrm{r} / \mathrm{min}$ for $10 \mathrm{~min}$ thrice to exclude the insoluble materials. Then, the solution was concentrated at $5000 \mathrm{r} / \mathrm{min}$ until the solution was clear to exclude the remaining GO. The material left was collected and dispersed in deionized water under supersonication. Then, the precipitate was collected, dispersed in deionized water, and dialysed to exclude the remaining salt and other small molecules.

\subsection{Synthesis of $\mathrm{MnO} 2 / \mathrm{R}-\mathrm{GO}-\mathrm{G}-\mathrm{GO}$}

$\mathrm{MnO}_{2} / \mathrm{R}-\mathrm{GO}-\mathrm{G}-\mathrm{GO}$ composites were prepared by $\mathrm{KMnO}_{4}$ and $\mathrm{GO}-\mathrm{G}-\mathrm{GO}$ under a hydrothermal condition. In a typical procedure, the prepared GO-G-GO (60 mg) was dispersed in $200 \mathrm{ml}$ of deionized water by supersonication to obtain a homogeneous dispersion. $\mathrm{KMnO}_{4}$ (180 mg) was then added to the above solution. After stirring for $4 \mathrm{~h}$ at $50^{\circ} \mathrm{C}$, the mixture was transferred to a Teflon-lined stainless-steel autoclave with a capacity of $250 \mathrm{ml}$ for hydrothermal treatment at $150{ }^{\circ} \mathrm{C}$ for $10 \mathrm{~h}$. After autoclaving, the sample was naturally cooled down to room temperature. The precipitate was separated by concentration, washed with distilled water, and dried in a vacuum at $60^{\circ} \mathrm{C}$ for $24 \mathrm{~h}$.

\subsection{Electrode preparation and characterization}

The working electrodes were fabricated by mixing the prepared powders with acetylene black (10wt\%) and binding with polyvinylidene fluoride (10wt\%), followed by adding a small amount of ethanol. About $2.5 \mathrm{mg}$ of the prepared mixture was pressed onto nickel foam $(1 \mathrm{~cm} \times 1 \mathrm{~cm})$ to make the electrode. Electrochemical studies were carried out in a three-electrode system. The prepared $\mathrm{MnO}_{2} / \mathrm{R}-\mathrm{GO}-\mathrm{G}-\mathrm{GO}$ composites were used as the working electrode, a platinum electrode $(1.5 \mathrm{~cm}$ $\times 1.5 \mathrm{~cm}$ ) was used as the counter electrode, and $\mathrm{Ag} / \mathrm{AgCl}$ electrode was used as the reference electrode. The electrolyte was $1 \mathrm{M} \mathrm{Na}_{2} \mathrm{SO}_{4}$ solution. $\mathrm{CV}$ and galvanostatic charge-discharge tests within different potential ranges were performed using CHI900C electrochemical working station system (Shanghai, China) at room temperature. Galvanostatic charge-discharge tests were performed using a land system (CT2001A Wuhan Xinuo Electronics and Technology Co., Ltd.).

\section{RESULTS AND ANALYSIS}

\subsection{External performances prediction}

The zeta potential spectrum of the as-synthesized GO-G-GO is shown in Fig. 1(e). The zeta potential of GO-G-GO solution was about $-50 \mathrm{mV}$, which meant that our material was homogeneously dispersed in deionized water, negatively charged, and highly stable. 

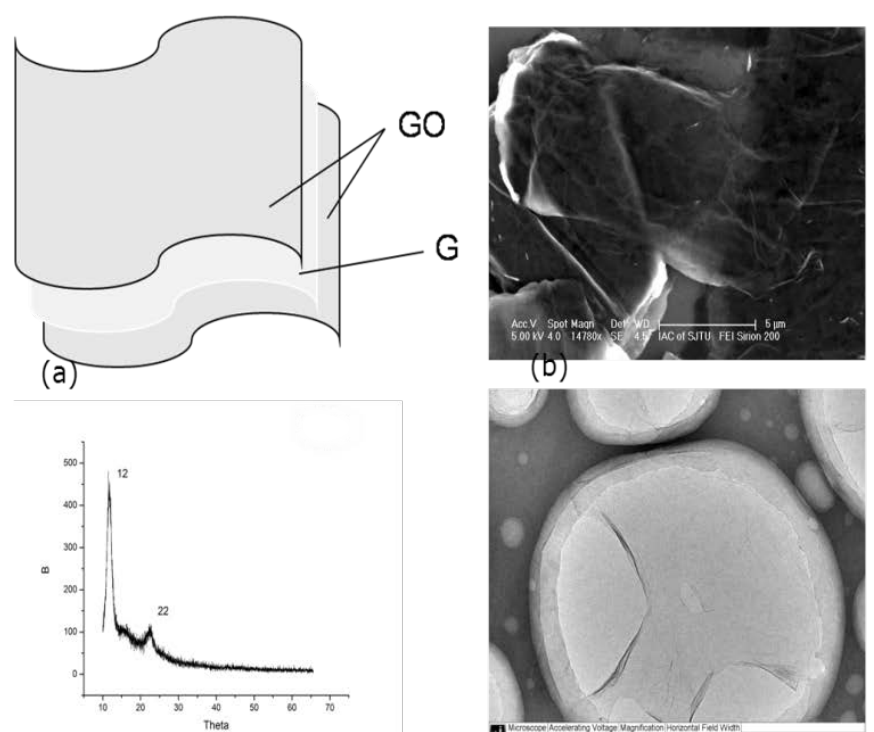

(c)
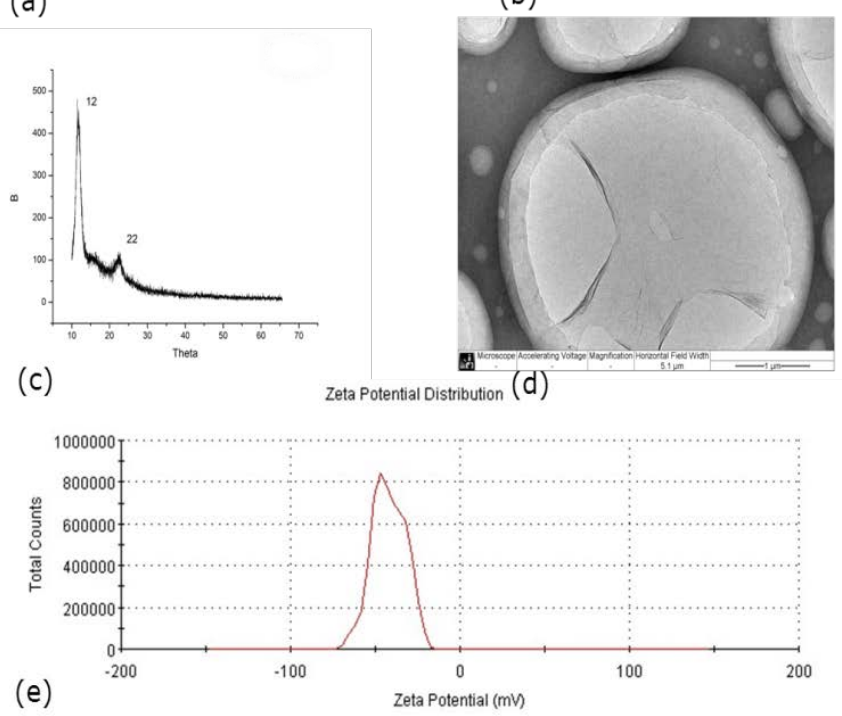

Fig. 1. Schematic of the structure of GO-G-GO material (a). SEM image of GO/G/GO dispersed in deionizer water (b). XRD patterns of prepared GO/G/GO (c). SEM image of as-synthesized GO (d). Zeta potential spectrum of GO-G-GO dispersed in deionizer water (e).

\subsection{XRD analysis}

The XRD spectrum of the as-synthesized GO/R-GO-G-GO/GO is shown in Fig. 1(c). The diffraction peak at $2 \theta=12.2^{\circ}$ differed from the GO peak at $2 \theta=10.0^{\circ}$ of the material synthesized by a modified Hummers method, and the peak at $2 \theta=22.4^{\circ}$ differed from the peak of graphite at $2 \theta=26.0^{\circ}$. No other clear sharp peaks were observed in the spectrum. Thus, this new kind of carbon material differed from GO and graphite.

The XRD spectrum of the as-prepared $\mathrm{MnO} 2 / \mathrm{R}-\mathrm{GO}-\mathrm{G}-\mathrm{GO}$ is shown in Fig. 2(b). The diffraction peaks at $2 \theta=26^{\circ}$ was the graphite peak. The XRD pattern can be indexed to monoclinic MnO2 (JCPDS no. 02-0909), whereas the XRD peaks of the MnO2/R-GO-G-GO composite overlapped with the diffraction peaks of $\mathrm{MnO} 2$ nanoparticles at $2 \theta=33.92^{\circ}, 25.53^{\circ}, 377.24^{\circ}$, $39.61^{\circ}, 41.27^{\circ}, 51.23^{\circ}, 53.74^{\circ}, 54.87^{\circ}, 56.01^{\circ}, 61.82^{\circ}$, and $64.95^{\circ}$. Apart from some differences in intensity, no characteristic peaks from other impurities were detected.
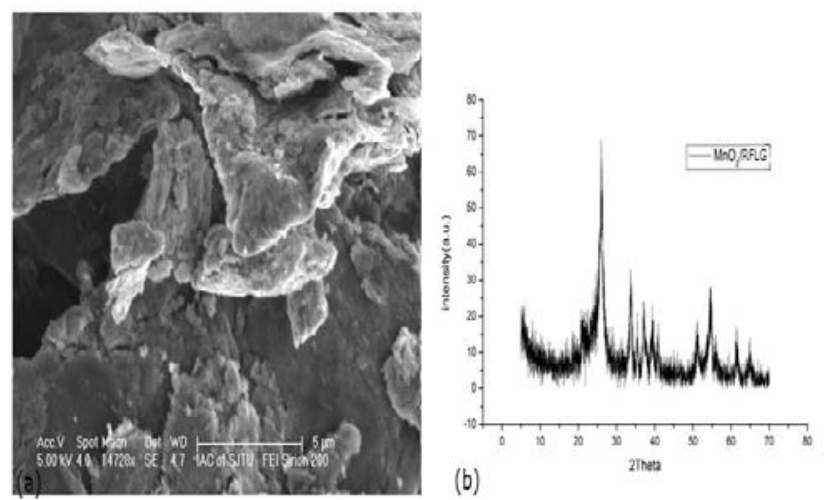

Fig. 2. (a) SEM image of $\mathrm{MnO}_{2} / \mathrm{R}-\mathrm{GO}-\mathrm{G}-\mathrm{GO}$ dried in air at $60^{\circ} \mathrm{C}$. (b) $\mathrm{XRD}$ patterns of as-prepared $\mathrm{MnO}_{2} / \mathrm{R}-\mathrm{GO}-\mathrm{G}-\mathrm{GO}$. 


\subsection{Morphology of the composites}

Fig. 1(b) is the SEM image of GO-G-GO dispersed in deionized water. The GO-G-GO nanoparticles were about $10 \mu \mathrm{m}$ long, whereas GO nanoparticles were only $1 \mu \mathrm{m}$ long (Fig. 1(d)), which obviously differed from GO. The GO-G-GO surface was very clear, which meant that no GO remained on the surface.

Fig. 2(a) is the SEM image of $\mathrm{MnO} / \mathrm{R}-\mathrm{GO}-\mathrm{G}-\mathrm{GO}$ powder dried in air at $60{ }^{\circ} \mathrm{C}$. This sample had a large microstructure and fixed with many nanoparticles, which obviously differed from GO. Thus, MnO2/R-GO-G-GO was successfully produced.

Electrochemical performance

The supercapacitor properties of the prepared MnO2/R-GO-G-GO composites were investigated by CV curves at different scan rates, as shown in Fig. 3(a). All scan rates had an almost rectangular shape. The mirror-like charge-discharge curves of $\mathrm{MnO} 2 / \mathrm{R}-\mathrm{GO}-\mathrm{G}-\mathrm{GO}$ showed its excellent electrical properties.
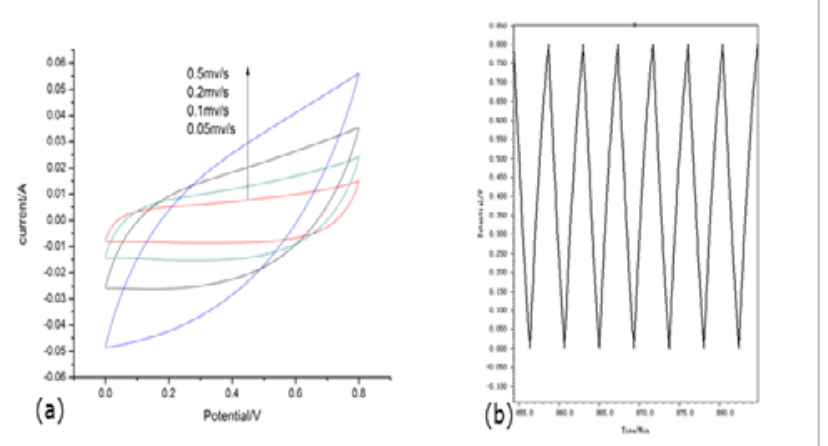

Fig. 3. (a) CV curves of MnO2/R-GO-G-GO composite at different scan rates (50, 100, 200, and $500 \mathrm{mV} / \mathrm{s}$ ).

(b) Galvanostatic charge-discharge curves of MnO2/RFLG composite at a current density of $1 \mathrm{~A} / \mathrm{g}$ in $1 \mathrm{M}$ $\mathrm{Na} 2 \mathrm{SO} 4$ solution.

As shown in Fig. 3(b), the symmetrical charge-discharge curve of MnO2/RFLG composite showed its excellent electrical properties as supercapacitor.

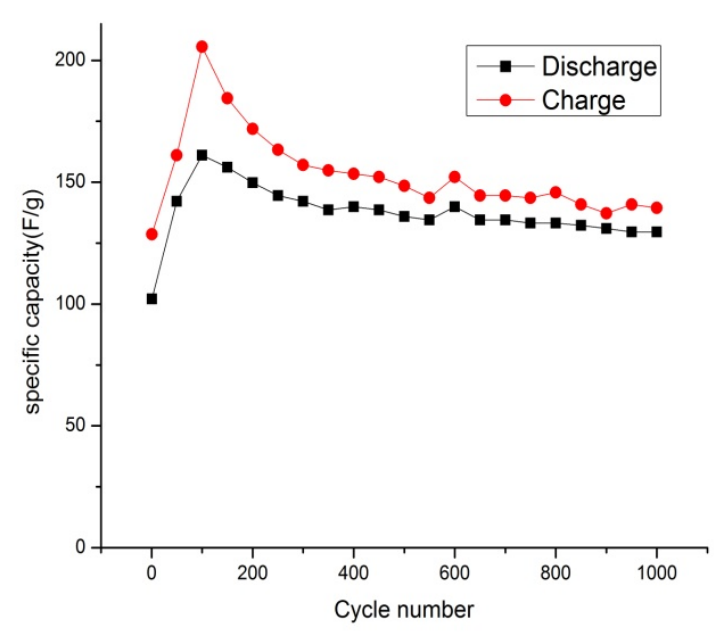

Fig. 4. Long-term cycle stability of $\mathrm{MnO} 2 / \mathrm{R}-\mathrm{GO}-\mathrm{G}-\mathrm{GO}$ composite electrode in $1 \mathrm{M}$ Na2SO4 aqueous solutions at a current density of $1 \mathrm{~A} / \mathrm{g}$.

Fig. 4 shows that our MnO2/R-GO-G-GO composite had excellent electrical properties. At the beginning of our test, the capacity increased may be because the mechanical structures of our materials had a random structure space. After 100 cycles, the discharge capacity increased to $160 \mathrm{~F} / \mathrm{g}$ and then decreased. After 200 cycles, the capacity was close to $145 \mathrm{~F} / \mathrm{g}$ and then slowly decreased. After 1000 cycles, the capacity was close to $140 \mathrm{~F} / \mathrm{g}$. 


\section{CONCLUSION}

Nanostructure GO-G-GO was successfully synthesized using a novel, easy, and environment-friendly method. A new kind of material consisting of one atomic layer of GO on the surface and a middle layer of graphene has never been reported. MnO2/R-GO-G-GO materials were also successfully synthesized by an easy, one-step method. CV and galvanostatic charge-discharge test results showed that the composite had good electrochemical reversibility in $1 \mathrm{M}$ Na2SO4 solution. MnO2/R-GO-G-GO also showed good cycle stability (140 F/g observed after 1000 cycles). Therefore, this new kind of material with good electrochemical performance can be used as materials for supercapacitors.

\section{REFERENCES}

[1] M. Endo, C. Kim, K. Nishimura, T. Fujino, K. Miyashita, Recent development of carbon materials for Li ion batteries, Carbon, 38 (2000) 183-197.

[2] S. Arepalli, H. Fireman, C. Huffman, P. Moloney, P. Nikolaev, L. Yowell, K. Kim, P. Kohl, C. Higgins, S. Turano, Carbon-nanotube-based electrochemical double-layer capacitor technologies for spaceflight applications, Jom, 57 (2005) 26-31.

[3] H.W. Kroto, J.R. Heath, S.C. O'Brien, R.F. Curl, R.E. Smalley, C 60: buckminsterfullerene, Nature, 318 (1985) 162-163.

[4] J.W. Wilder, L.C. Venema, A.G. Rinzler, R.E. Smalley, C. Dekker, Electronic structure of atomically resolved carbon nanotubes, Nature, 391 (1998) 59-62.

[5] K.S. Novoselov, A.K. Geim, S. Morozov, D. Jiang, Y. Zhang, S. Dubonos, I. Grigorieva, A. Firsov, Electric field effect in atomically thin carbon films, science, 306 (2004) 666-669.

[6] R. Nair, P. Blake, A. Grigorenko, K. Novoselov, T. Booth, T. Stauber, N. Peres, A. Geim, Fine structure constant defines visual transparency of graphene, Science, 320 (2008) 1308-1308.

[7] S. Stankovich, D.A. Dikin, G.H. Dommett, K.M. Kohlhaas, E.J. Zimney, E.A. Stach, R.D. Piner, S.T. Nguyen, R.S. Ruoff, Graphene-based composite materials, Nature, 442 (2006) 282-286.

[8] K. Novoselov, A.K. Geim, S. Morozov, D. Jiang, M. Katsnelson, I. Grigorieva, S. Dubonos, A. Firsov, Two-dimensional gas of massless Dirac fermions in graphene, Nature, 438 (2005) 197-200.

[9] F. Liu, P. Ming, J. Li, Ab initio calculation of ideal strength and phonon instability of graphene under tension, Physical Review B, 76 (2007) 064120.

[10] C.A. Marianetti, H.G. Yevick, Failure mechanisms of graphene under tension, Physical review letters, 105 (2010) 245502.

[11] A.A. Balandin, S. Ghosh, W. Bao, I. Calizo, D. Teweldebrhan, F. Miao, C.N. Lau, Superior thermal conductivity of single-layer graphene, Nano letters, 8 (2008) 902-907.

[12] A.A. Balandin, Thermal properties of graphene and nanostructured carbon materials, Nature materials, 10 (2011) 569-581.

[13] K.F. Mak, M.Y. Sfeir, Y. Wu, C.H. Lui, J.A. Misewich, T.F. Heinz, Measurement of the optical conductivity of graphene, Physical review letters, 101 (2008) 196405.

[14] M. Bruna, S. Borini, Optical constants of graphene layers in the visible range, Applied Physics Letters, 94 (2009) 031901.

[15] H. Chen, M.B. Müller, K.J. Gilmore, G.G. Wallace, D. Li, Mechanically strong, electrically conductive, and biocompatible graphene paper, Advanced Materials, 20 (2008) 3557-3561. 